\title{
Computer Vision for the Remote Sensing of Atmospheric Visibility
}

\author{
Raouf Babari, Nicolas Hautière, Eric Dumont \\ Université Paris-Est, IFSTTAR, IM, LEPSIS \\ 58 boulevard Lefebvre \\ F-75015 Paris \\ nicolas.hautieredifsttar.fr
}

\author{
Jean-Pierre Papelard, Nicolas Paparoditis \\ Université Paris-Est, IGN, MATIS \\ 73 avenue de Paris \\ F-94160 Saint-Mandé \\ nicolas.paparodits@ign. fr
}

\begin{abstract}
Atmospheric visibility distance is a property of the atmosphere, which can be remotely sensed by computer vision. In this aim, a non-linear mapping function between the atmospheric visibility distance and the contrast in images must be estimated. The function depends on the scene depth distribution as well as on the radiometry of the scene. In order to calibrate and deploy such camera-based atmospheric visibility estimations, we present two methods which aim at computing the scene depth distribution and the radiometry of the scene beforehand. The scene depth is recovered by registering a full $3 D$ model of the environment in the frame of the camera. The radiometry of the scene is partly recovered by looking at the temporal correlation between the variation of pixels intensity and the variation of the sky luminance estimated by a luminance meter oriented toward the North direction. Based on clear-sky models, it is demonstrated that such a process detects a set of pixels, which include pixels belonging to North-oriented Lambertian surfaces. This finding leads to a simplified way of detecting Lambertian surfaces without any additional luminance meter. Good results obtained experimentally prove that such techniques are relevant to estimate the atmospheric visibility distance.
\end{abstract}

\section{Introduction}

Atmospheric visibility distance is a crucial information for transport safety as well as a good evidence of air pollution. Electro-optical sensors such as transmitometers or scatterometers have been developed to measure this environmental parameter. Transmitometers are reliable but are very expensive, which explains why they are deployed at critical places like major airports. The reliability of scatterometers is sometimes questionable but these instruments are still quite expensive. Cameras are multi-functional sensors, which are massively deployed in urban cities, along road networks or in tourist attractions for safety and security reasons. These sensors are potentially good environmental sensors and allow estimating the meteorological visibility distance.

Different methods have been developed to build camerabased visibility meters. A first family of method detects the most distant visible picture elements in the scene. These methods only need a good geometrical calibration of the camera to be operated. Nevertheless, they are restricted to the depth distribution of objects which are in the field of view of the camera. In the field of road safety, the road surface is considered and the range of considered distances is lower than $1000 \mathrm{~m}[5,8]$. In the field of meteorological observation, higher ranges are considered by using points of interests like hills or mountains [4].

A second of family of methods seeks a mapping function between a reference measurement obtained by an external data source, e.g. a visibility meter, and an imagebased visibility descriptor. High-frequency filters such as a Sobel gradient or a high-pass filter based on a fast fourier transform are commonly used [15]. These basic descriptors being sensitive to illumination variations, different methods were proposed to cope with this situation. [14] proposed to use a homomorphic filter or a Haar function in addition to the high-pass filter in order to reduce the effects of nonuniform illumination. [3] proposed a visibility descriptor which is robust to illumination variations by making use of Lambertian surfaces only. However, the method to select the Lambertian surfaces is superfificially justified.

The methods differ also by the way the mapping function is built. In the field of atmospheric environment, a linear fitting of data is generally performed, but such approaches are not valid anymore in case of shorter visibility ranges. Recently, a model-driven approach has been proposed [2]. The calibration of the method is made easier by introducing a physics-based model and a simplified depth distribution model of the scene. This method allows dealing with a full range $(0-15000 \mathrm{~m})$ of visibility distances by applying a non-linear fitting to the data. However, the obtention of the depth distribution model of the scene still need a pair 
of fog images, which still hinder the deployment of such a method.

In this paper, we address two of the aforementioned problems. First, we propose to select the Lambertian surfaces in a scene by computing the temporal correlation between the variation of a pixel intensity and the variation of the luminance of a sky element. This empirical method is compared to an analytical method and validated in a controlled environment. Second, we propose to compute the depth distribution by registering the scene with a full $3 \mathrm{D}$ model of the environment.

The paper is organized as follows. First, Koschmieder's theory on the visual appearance of objects in the atmosphere is recalled as well as the model-driven approach proposed in [2] to estimate the visibility by camera. Second, our method of Lambertian surfaces selection is presented and justified. Third, we show how we propose to compute a depth distribution model, based on existing geographical databases. Fourth, an experimental evaluation of the work carried out is proposed. Finally, results are discussed and perspectives are drawn.

\section{Vision through the Atmosphere}

\subsection{Koschmieder's Theory}

The attenuation of luminance through the atmosphere was studied by Koschmieder [16], who derived an equation relating the extinction coefficient of the atmosphere $\beta$, which is the sum of the scattering coefficient and of the absorption coefficient, the apparent luminance $L$ of an object located at distance $d$, and the luminance $L_{0}$ measured close to this object:

$$
L=L_{0} e^{-\beta d}+L_{\infty}\left(1-e^{-\beta d}\right)
$$

(1) indicates that the luminance of the object seen through fog is attenuated by $e^{-\beta d}$ (Beer-Lambert law); it also reveals a luminance reinforcement of the form $L_{\infty}\left(1-e^{-\beta d}\right)$ resulting from daylight scattered by the slab of fog between the object and the observer, the so-called airlight. $L_{\infty}$ is the atmospheric luminance.

On the basis of this equation, Duntley developed a contrast attenuation law [16], stating that a nearby object exhibiting contrast $C_{0}$ with the fog in the background will be perceived at distance $d$ with the following contrast:

$$
C=\left[\frac{L_{0}-L_{\infty}}{L_{\infty}}\right] e^{-\beta d}=C_{0} e^{-\beta d}
$$

This expression serves to base the definition of a standard dimension called meteorological visibility distance $V$, i.e. the greatest distance at which a black object $\left(C_{0}=-1\right)$ of a suitable dimension can be seen on the horizon, with the threshold contrast set at 5\% [6]. It is thus a standard parameter that characterizes the opacity of a fog layer. This definition yields the following expression:

$$
V \approx \frac{3}{\beta}
$$

\subsection{Contrast of Lambertian Targets}

Assuming a linear response function of the camera, the intensity $I$ of a distant point located at distance $d$ in an outdoor scene is given by Koschmieder's model (1):

$$
I=R e^{-\beta d}+A_{\infty}\left(1-e^{-\beta d}\right)
$$

where $R$ is the intrinsic intensity of the pixel, i.e. the intensity corresponding to the intrinsic luminance value of the corresponding scene point and $A_{\infty}$ is the background sky intensity. Two points located at roughly the same distance $d_{1} \approx d_{2}=d$ with different intensities $I_{1} \neq I_{2}$ form a distant target whose normalized contrast is given by:

$$
C=\frac{I_{2}-I_{1}}{A_{\infty}}=\left[\frac{R_{2}-R_{1}}{A_{\infty}}\right] e^{-\beta d}=C_{0} e^{-\beta d}
$$

In this equation, the contrast $C$ of a target located at distance $d$ depends on $V=\frac{3}{\beta}$ and on its intrinsic contrast $C_{0}$. If we now assume that the surface of the target is Lambertian, the luminance $L$ at each point $i$ of the target is given by:

$$
L=\rho_{i} \frac{E}{\pi}
$$

where $E$ denotes the global illumination and $\rho_{i}$ denotes the albedo at $i$. Moreover, it is a classical assumption to set $L_{\infty}=\frac{E}{\pi}$ so that (5) finally becomes:

$$
C=\left(\rho_{2}-\rho_{1}\right) e^{-\beta d} \approx\left(\rho_{2}-\rho_{1}\right) e^{-\frac{3 d}{V}}=\Delta \rho e^{-\frac{3 d}{V}}
$$

Consequently, the contrast of a distant Lambertian target only depends on its physical properties and on its distance to the sensor and on the meteorological visibility distance, and no longer on the illumination. These surfaces are robust to strong illumination variations in the computation of the contrast in the scene.

\section{The Model-Driven Approach}

Let us consider an outdoor scene where targets are distributed continuously at increasing distances from the camera. Let us denote $\phi$ the probability density function of observing a contrast $C$ in the scene:

$$
\mathbb{P}(C<X \leq C+\mathrm{d} C)=\phi(C) \mathrm{d} C
$$

The expectation of the contrast $m$ in the image is expressed as:

$$
m=\mathbb{E}[C]=\int_{0}^{1} C \phi(C) \mathrm{d} C
$$




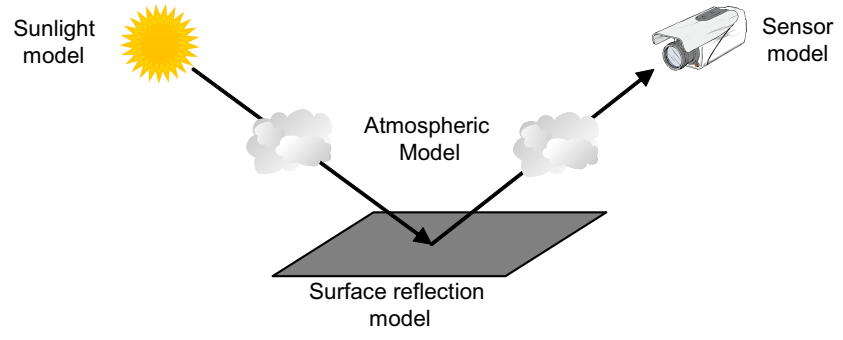

Figure 1. Components of the radiometric sensing problem.

Based on (7), $C$ is a random variable which depends of the two random variables $d$ and $\Delta \rho$. These two variables are assumed to be independent, which allows expressing (9) as:

$$
m=\mathbb{E}[\Delta \rho] \mathbb{E}\left[e^{-\frac{3 d}{V}}\right]=\overline{\Delta \rho} \int_{0}^{+\infty} \psi(d) e^{-\frac{3 d}{V}} \mathrm{~d} d
$$

where $\overline{\Delta \rho}$ denotes the mean albedo difference between the objects in the scene and $\psi$ denotes the p.d.f. of there being an object at the distance $d$ in the scene. To compute $m$, a realistic expression for the density of objects $\psi$ in the scene is needed, as well as a map of the albedo of the Lambertian surfaces [2].

\section{Classification of Lambertian Surfaces}

\subsection{Principle}

Classifying the objects in a scene image which are likely to follow Lambert's law is a difficult problem. Indeed, during the process of image formation, four components interact with each other (see Fig. 1), which makes it difficult to separate the numerous physical variables. The first component is the sunlight. The second component is the atmosphere. The third component is the nature of the surfaces. The third component is the response function of the camera. To solve this complex problem, we estimate the surface properties in clear-sky conditions and rely on the analysis of time-series of pixel intensities during the motion of the sun $[11,1,10]$.

\subsection{Materials}

\subsubsection{Sky Model}

Perez's sky model [18] describes the luminance of any arbitrary sky element as a function of its elevation, and its relative orientation with respect to the sun [13]. Consider the illustration in Fig. 2, the relative luminance $l_{p}$ of a sky element is a function of its zenith angle $\theta_{p}$ and the angle $\gamma_{p}$ with the sun:

$$
\begin{aligned}
l_{p} & =\left[1+a\left(b / \cos \theta_{p}\right)\right] \cdot\left[1+c \exp \left(d \gamma_{p}\right)+e \cos ^{2} \gamma_{p}\right] \\
& =f\left(\theta_{p}, \gamma_{p}\right)
\end{aligned}
$$

where the 5 parameters $(a, b, c, d, e)$ specify the current atmospheric conditions.

The atmosphere is usually not transparent, especially in case of fog or haze or pollution. In our case, we aim at estimating the Koschmieder's model parameters, knowing surface properties. We thus assume that the atmosphere is clear and thus use input images which have been grabbed in presence of fine weather. For clear skies, the parameters of Perez's sky model take on the following values: $a=-1$, $b=-0.32, c=10, d=-3, e=0.45$.

The model expresses the absolute luminance $L_{p}$ of a sky element as a function of another arbitrary reference sky element. For instance, if the zenith luminance $L_{z}$ is known, then:

$$
L_{p}=L_{z} \frac{f\left(\theta_{p}, \gamma_{p}\right)}{f\left(0, \theta_{s}\right)}
$$

where $\theta_{s}$ is the zenith angle of the sun. Many equations for the zenith luminance are proposed in the literature [7]. For a clear sky, Krochman's model is as following [12]:

$$
L_{z}=0.1+0.063 \theta_{s}+0.001 \theta_{s}\left(\theta_{s}-30\right) e^{0.0346\left(\theta_{s}-68\right)}
$$

where $\theta_{s}$ is expressed in degrees. By combining (11),(12) and (13), we are able to express the absolute luminance of any arbitrary reference sky element.

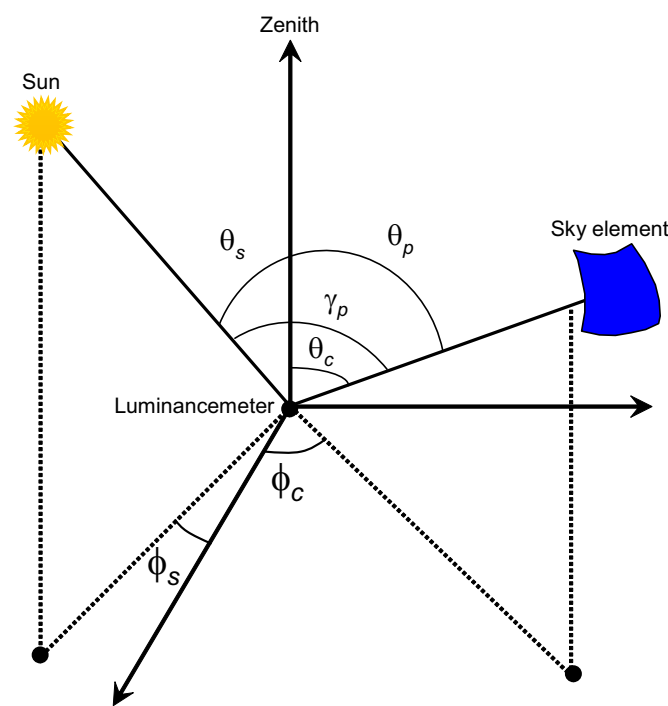

Figure 2. Geometry of Perez's sky model. The sun direction is given by $\left(\theta_{s}, \phi_{s}\right)$, the sky element direction is given by $\left(\theta_{c}, \phi_{c}\right)$ and the angle between the sun and the sky element is $\gamma_{p}$. 


\subsubsection{Light Transport Model}

The radiance at a point $\vec{x}$ in a scene is given by the light transport equation:

$$
L_{o}\left(\vec{x}, \vec{\omega}_{o}\right)=\int_{\Omega} \operatorname{brdf}\left(\vec{x}, \vec{\omega}_{o}, \vec{\omega}_{i}\right) \cdot E_{i}\left(\vec{x}, \overrightarrow{\omega_{i}}\right) \cdot(\vec{n}(\vec{x}) \cdot \vec{l}(\vec{x})) \mathrm{d} \omega_{i}
$$

where $L_{o}(\vec{x})$ denotes the outgoing radiance from point $\vec{x}$, $E_{i}(\vec{x})$ the input radiance at point $\vec{x}$, brdf the bidirectional reflectance distribution function of the material at point $\vec{x}$. We want to detect the Lambertian surfaces, hence a diffuse brdf can be used in the light transport equation:

$$
L_{o}(\vec{x})=\frac{\rho}{\pi} \sum_{i=1}^{N} E_{i, n}(\vec{x}) \cdot(\vec{n}(\vec{x}) \cdot \vec{l}(\vec{x}))
$$

As we restrict ourselves to an outdoor scene during daytime, we assume that the illumination can be divided into two terms - direct light from the sun $E_{d}$, and ambient light $E_{a}$ from the sky. The light transport equation can again be simplified:

$$
L_{o}(\vec{x})=\frac{\rho}{\pi}\left(E_{a}+E_{d} \cdot(\vec{n}(\vec{x}) \cdot \vec{l}(\vec{x}))\right)
$$

\subsubsection{Sensor Model}

The outgoing radiance $L_{o}(\vec{x})$ from point $\vec{x}$ is sampled from an input image of the scene by the sensor. The gray level at each point will the direct radiance of the point scaled by a constant if the camera has a linear response. This scaling constant is denoted $k$, while the unit-less value provided by the camera is $G(\vec{x})$ :

$$
G(\vec{x})=k \cdot \frac{\rho}{\pi}\left(E_{a}+E_{d} \cdot(\vec{n}(\vec{x}) \cdot \vec{l}(\vec{x}))\right)
$$

We have to choose a reasonable value for the gain of the camera $k$. The camera may be adapted to the sunlight or to the ambient light. Let us test these two assumptions.

First, we assume that the camera is adapted to the sunlight, so that $k \propto \frac{1}{E_{d}}$. (17) becomes:

$$
G(\vec{x}) \propto \frac{\rho}{\pi}\left(\frac{E_{a}}{E_{d}}+(\vec{n}(\vec{x}) \cdot \vec{l}(\vec{x}))\right)
$$

A reasonable assumption, consistent with models from [7], consists in assuming that the ambient light is proportional to the sunlight, i.e. $\frac{E_{a}}{E_{d}}=c$. (18) thus becomes:

$$
G(\vec{x}) \propto \frac{\rho}{\pi}(c+\vec{n}(\vec{x}) \cdot \vec{l}(\vec{x}))
$$

Second, we assume that the camera is adapted to the ambiant light, so that $k \propto \frac{1}{E_{a}}$. (17) thus becomes:

$$
G(\vec{x}) \propto \frac{\rho}{\pi}\left(1+\frac{1}{c}(\vec{n}(\vec{x}) \cdot \vec{l}(\vec{x}))\right)
$$

\subsubsection{Modeling the Illumination with the Motion of the Sun}

The direction of the sunlight, $\vec{l}(\vec{x})$, at time $t$ and the surface normal of a point $i, \vec{n}(\vec{x})$, can be expressed in Cartesian coordinates as in the following equation where $\phi$ 's are the azimuth angles and $\theta$ 's are the elevation angles.

$$
\begin{aligned}
\vec{l}(\vec{x}) & =\left[\cos \theta_{t} \cos \phi_{t}, \cos \theta_{t} \sin \phi_{t}, \sin \theta_{t}\right]^{T} \\
\vec{n}(\vec{x}) & =\left[\cos \theta_{i} \cos \phi_{i}, \cos \theta_{i} \sin \phi_{i}, \sin \theta_{i}\right]^{T}
\end{aligned}
$$

The scalar product is then:

$$
\vec{n}(\vec{x}) \cdot \vec{l}(\vec{x})=\cos \theta_{t} \cos \theta_{i} \cos \left(\phi_{t}-\phi_{i}\right)+\sin \theta_{t} \sin \theta_{i}
$$

According to [10], $\vec{l}(\vec{x})$ and $\vec{n}(\vec{x})$ are rotated so that $\theta_{t}=$ 0 without any loss of generality, which implies that (23) becomes:

$$
\vec{n}(\vec{x}) \cdot \vec{l}(\vec{x})=\cos \theta_{i}^{\prime} \cos \left(\phi_{t}-\phi_{i}\right)
$$

According to (24), the lighting variation at a point due to the sun over time is a sinusoidal function with the scale and the phase being the parameter. Combining (19) or (20) with (24) allows obtaining the intensity of a pixel belonging to a Lambertian object as a sinusoidal function with amplitude and phase as parameters:

$$
G(\vec{x}) \propto \frac{\rho \delta}{\pi} \cos \left(\phi_{t}-\phi_{i}\right)
$$

where $\delta=\cos \theta_{i}^{\prime}$ if the camera is adapted to the sunlight and $\delta=c \cos \theta_{i}^{\prime}$ if the camera is adapted to the ambient light. If we only consider surfaces whose normal is North-oriented, we have $\phi_{i}=0$ and obtain:

$$
G(\vec{x}) \propto \frac{\rho \delta}{\pi} \cos \phi_{t}
$$

\subsection{Method}

The linear coefficient of correlation of Bravais-Pearson is computed between the measured gray level taken from the image $G_{t}$ and the expected gray level profile over time for each pixel $g_{t}$.

$$
r_{p}=\frac{\sum_{t}\left(G_{t}-\bar{G}_{t}\right) \cdot\left(g_{t}-\bar{g}_{t}\right)}{\sqrt{\sum_{t}\left(G_{t}-\bar{G}_{t}\right)^{2}} \cdot \sqrt{\sum_{t}\left(g_{t}-\bar{g}_{t}\right)^{2}}}
$$

where $\bar{G}_{t}$ and $\bar{g}_{t}$ are the sample means of $G_{t}$ and $g_{t} . r_{p}$ is computed for each pixel to give the "Lambertian confidence map". When the correlation $r_{p}$ obtained for a pixel is close to 1 , the pixel is supposed to have Lambertian behavior, because of the expected linearity between the measured gray 


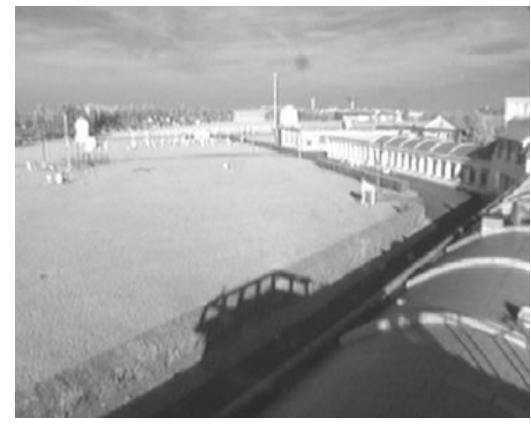

(a)

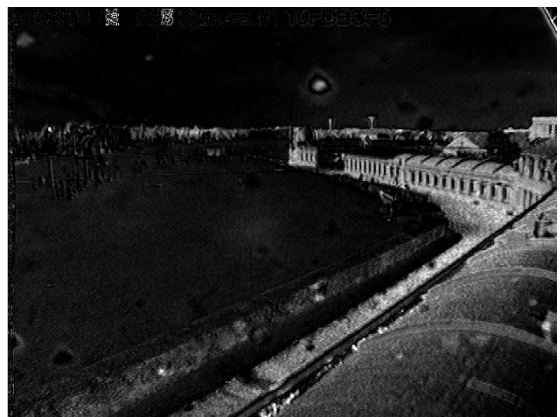

(b)

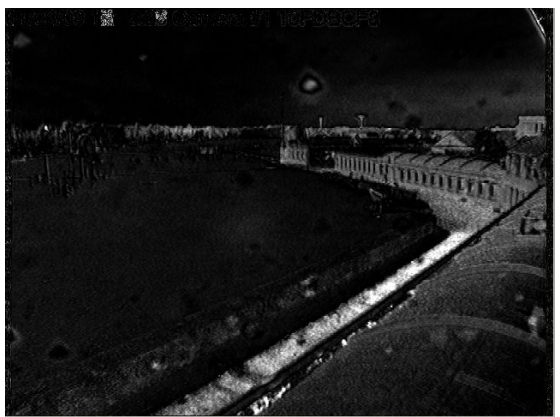

(c)

Figure 3. Lambertian confidence map: (a) the test site; (b) the map obtained using the luminance profile of the sky measured by an additional luminancemeter; (c) the map predicted by a sinusoidal function which accounts for the motion the sun.

level $G_{t}$ and the expected one $g_{t}$. The behavior is otherwise unknown. In short, the temporal correlation between the expected and the measured gray level, is an indicator of the surface to be Lambertian or not. The expected gray level profile has now to be chosen. We have tested two different options:

- A first method proposed by [3] consists in using the profile of luminance of a sky region measured by an additional luminance meter shown in Fig. 4. By using the MATILDA database [9], the Lambertian confidence map shown in Fig. 3(a) is obtained. This luminance profile, which was experimentally obtained, can be roughly assimilated to a sinusoidal function of the time. Indeed, we modeled in section 4.2.1 the luminance of any arbitrary sky element by combining Perez's sky model and Krochmann's zenith luminance model. We computed this model with respect to the sun $\theta_{s}$ angle variation. We chose a relevant sky element, i.e. the sky element observed by the luminance meter. This one is oriented towards the North direction

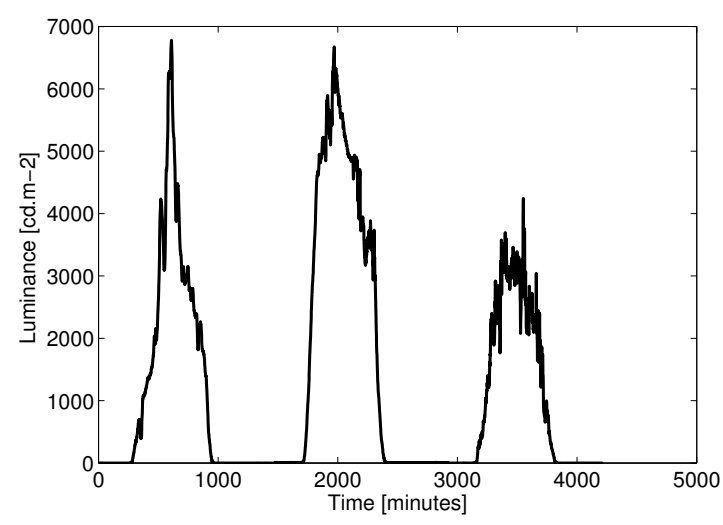

Figure 4. Luminance profile measured by a luminance meter oriented toward the North with a site angle of $40^{\circ}$.

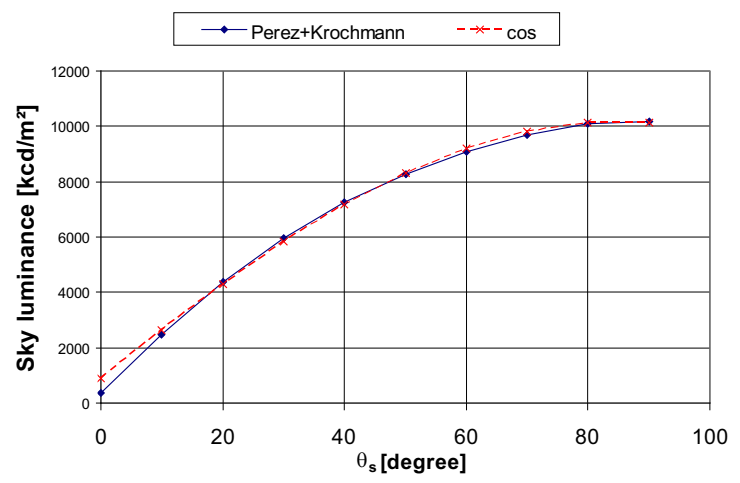

Figure 5. Theoretical luminance profile of a sky element oriented toward the North direction with a site angle of $40^{\circ}$ obtained by combining Perez's sky model and Kochmann's zenith luminance model. A sinusoidal curve is plotted to show the similarity of both profiles.

and has a site angle of $40^{\circ}$, i.e. $\theta_{p}=50^{\circ}$ (see Fig. 2). The obtained curve is plotted in Fig. 5. We also plot a sinusoidal function. As one can see, both curves are roughly the same shape.

- A second method consists in choosing the expected gray-level profile $g_{t}$ as a sinusoidal function according to (26). This leads to the Lambertian confidence map shown in Fig. 3(b).

Whereas both methods are not based on the same theoretical foundations, the mathematical functions are the same which means that they should give approximately the same results. This is confirmed experimentally by comparing Figs. 3(a)\&(b), where the same areas of the scene are considered as including North-oriented Lambertians surfaces. Nevertheless, the map obtained with the sinusoidal model seems to be a little more noisier. 
(a)
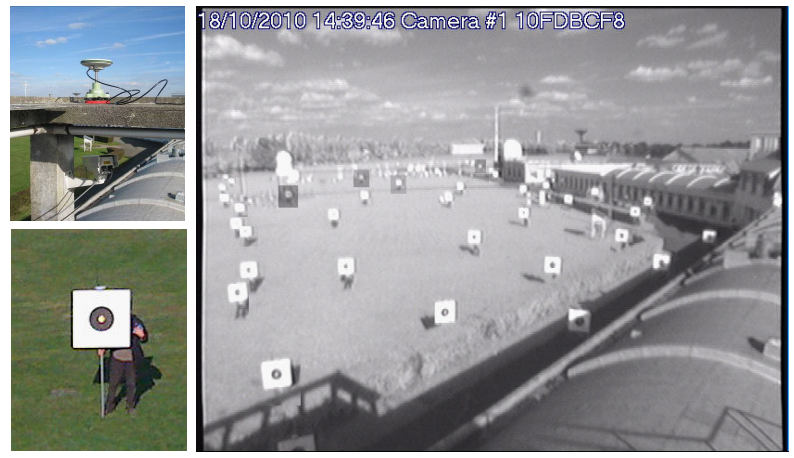

(c)

Figure 6. Calibration of the camera: (a) estimation of the geolocation of the camera by a GPS receiver; (b) target used for geometrical calibration; (c) different positions of the target to calibrate the camera.

\section{Estimation of a Scene Depth Distribution}

To solve (10), we need a map of Lambertian pixels as well as a depth distribution of the scene. The previous section was dealing with the estimation of the Lambertian objects in the scene. In this section, we deal with the estimation of the depth distribution $\psi$.

\subsection{Principle}

This is difficult problem which cannot be solved without any additional information. [19] proposed some a priori depth distributions in natural or man-made scenes which are Gaussian distributions. These models are too much approximative in our case. The camera does not move which does not allow to use structure from motion algorithms. Since we are dealing with visibility problems, fog can be used to infer a scene structure. [17] used a pair of fog images grabbed in different fog conditions to compute an approximate 3D model of a city model. This method is used in [2] to compute a scene depth model and derive the distribution model $\psi$ to be used in (10). A new possibility which has been explored consists in using an existing 3D model of the environment, like in [4]. However, they only register a few interest points and obtain a sparse depth map. In this paper, we propose to register a full 3D model of the environment.

\subsection{Implementation}

First, the intrinsic and extrinsic parameters of the camera have to be calibrated and its absolute pose must be measured. In this aim, the geolocation of camera has been estimated using an accurate GPS receiver (see Fig. 6(a)). Then, a series of 35 images with a target (see Fig. 6(b)) equipped with a GPS antenna was grabbed. The image in Fig. 6(c) shows the different positions of the targets. These different information allow estimating the intrinsic parameters (in particular the distortion parameters) of the camera in an ab-

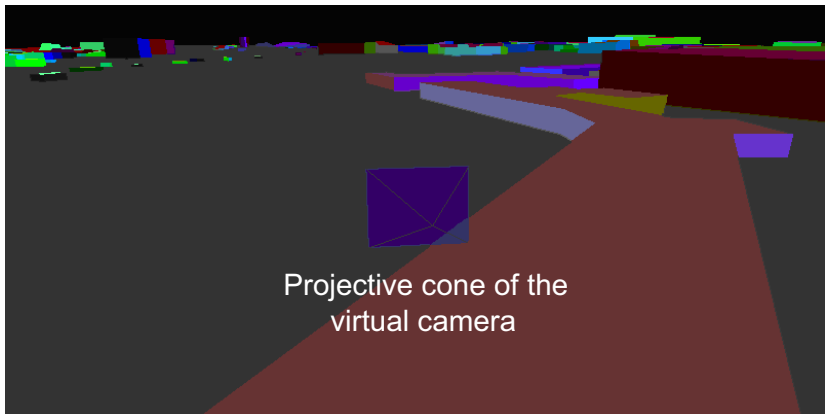

Figure 7. 3D model of the environment (DTM+3D buildings) projected in the image space of the camera. The color corresponds to the id of the objects.

solute geographic reference frame. A LOD1 3D model of the environment of one meter accuracy, constructed from a DTM and the extrusion of a national data basis of 3D building gutter lines, is then projected in image space to construct a depth map (see Fig. 7).

From this 3D view, a dense depth map can be reconstructed. A depth map of the scene is shown in Fig. 8), where the actual characteristics of the camera which were previously estimated, are used. A depth histogram $H_{d}$ of Fig. 8 is deduced and is shown in Fig. 9. An exponential distribution is fitted to $H_{d}$ and is overlaid in red in Fig. 9. We assume that the distribution $\psi$ of (10) follows the same model. Because $H_{d}$ dos not take into account the Lambertian surfaces, this assumption means that we assume a uniform distribution of Lambertian surfaces with respect to the scene depth.

\section{Experimental Evaluation}

In this section, an experimental evaluation of our visibility estimation method on the MATILDA public database [9]

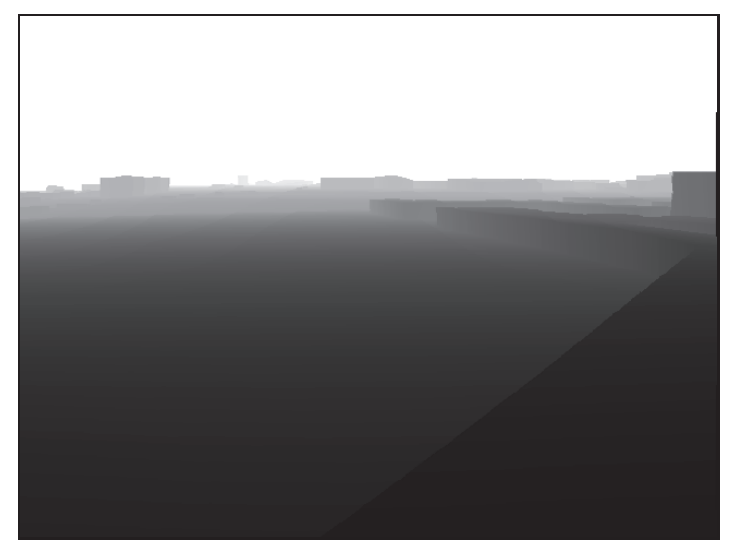

Figure 8. Dense depth map reconstructed from the 3D model of the environment. 
is carried out. In section 4, we have proposed two methods to detect Lambertian surfaces in an outdoor scene. In section 5, we have proposed a method to compute a depth distribution $H_{d}$ of a scene using a 3D GIS, which is assumed to follow an exponential distribution. Based on these results, we are able to solve (10) using the mathematical solutions provided in [2]:

$$
m=\frac{1}{N} \sum_{i, j} \frac{\nabla_{i, j} r_{p_{i, j}}}{A_{\infty}} \approx \frac{\eta \overline{\Delta \rho}}{\eta+\frac{3}{V}}
$$

where $\nabla$ denotes the gradient operator and $r_{p}$ the confidence that the pixel is Lambertain obtained by linear correlation. $\eta$ is obtained by fitting the data of MATILDA with a low visibility distance $(<1000 \mathrm{~m})$ with ground truth data. The model is then extrapolated for the higher visibility distances following the methodology proposed in [2]. The adjusted model is plotted in Fig. 10.

Results are detailed in Table 1 and give the mean relative error on the MATILDA database for the different applications proposed in [2]. The new results are on the last line of the table and were obtained by choosing $\psi$ as an exponential distribution and by using a sinusoidal function to determine the confidence Lambertian map, i.e the map shown in Fig. 3(c). The obtained results still stlightly improve the state of the art for all ranges of visibility compared to existing methods, except the range 0-10,000 dealing with air quality. This can be explained by the few outliers above the model in Fig. 10.

These results allow to validate two assumptions. First, an additional luminance meter is not mandatory to compute a relevant confidence map of Lambertian surfaces. A sinusoidal function, applied with images acquired in clear sky conditions, is a good substitute. Second, the type of depth distribution can be guessed by registering a 3D GIS in the frame of the camera image. These two results are very important in order to calibrate camera visibility meters without any fog episodes.

\section{Conclusion and Perspectives}

Atmospheric visibility distance is a property of the atmosphere, which can be remotely sensed by computer vision. In this aim, a non-linear mapping function between the atmospheric visibility distance and the contrast in images must be estimated. This function depends on the scene depth distribution as well as on the radiometry of the scene. In order to estimate a scene depth distribution, we propose to register a three dimensional geographical information system (GIS) in the frame of the camera. This method allows guessing a model of the actual distribution. In our experimental tests, this was enough to estimate correctly the visibility distance. However, in this work we only used the histogram of depth. In the future, it should be interesting

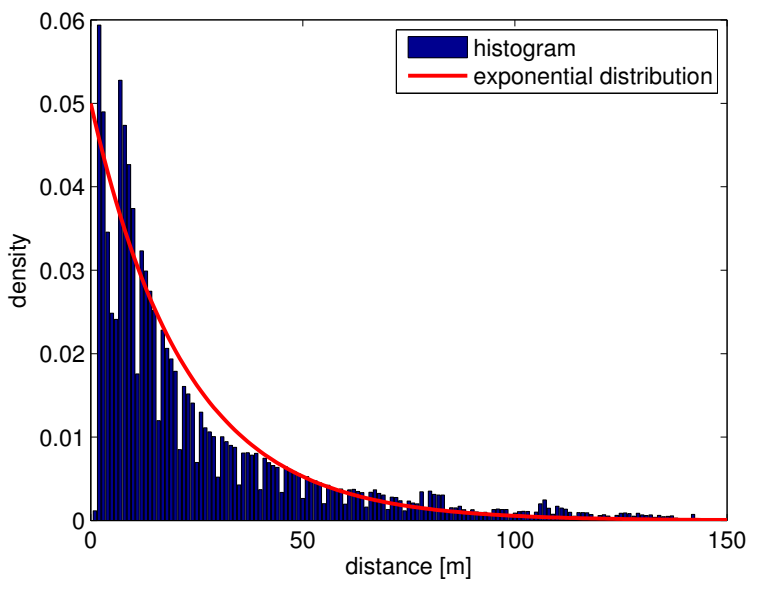

Figure 9. Depth distribution of the test scene. The histogram of the depth map is plotted using bars. An exponential distribution is adjusted to these data and is shown in red color.

to use this kind of depth map to implement other visibility monitoring methods like the methods proposed by Bäumer et al. [4] dedicated to meteorological observations or the method proposed by Hautière et al. [8] dedicated to road safety. In this aim, an automatic registration of the depth image in the camera frame should be added to improve the robustness of the method. Then, the radiometry of the scene is partially recovered by looking at the temporal correlation between the variation of pixels intensity and a sinusoidal function. Based on clear-sky models, it is demonstrated that such a process detects a set of pixels, which corresponds to North-oriented Lambertians surfaces. This result is con-

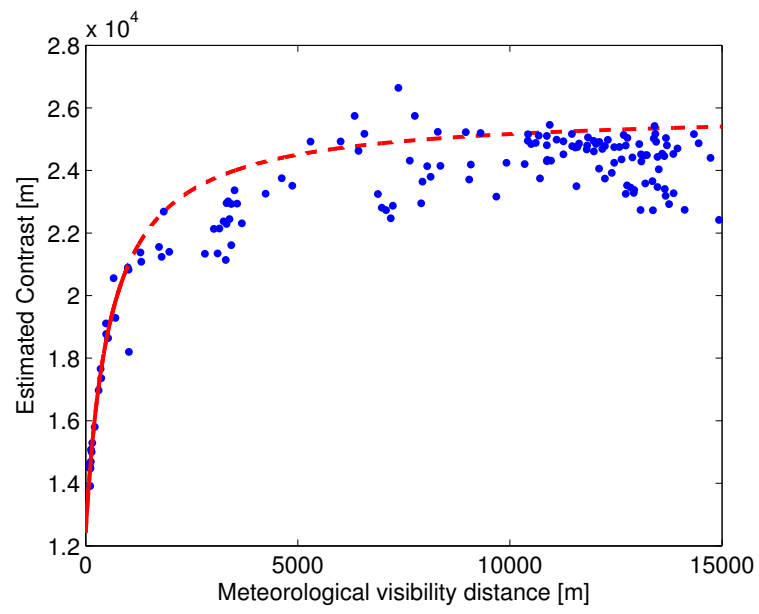

Figure 10. Data fitting with the exponential distribution model. A few outliers can be seen above the model in the range 5,000-10,000 which explain a lower accuracy of the model in this range. 


\begin{tabular}{cccccc}
\hline Application & $\begin{array}{c}\text { Ground Transport } \\
\text { Safety }\end{array}$ & $\begin{array}{c}\text { Air Transport } \\
\text { Safety }\end{array}$ & $\begin{array}{c}\text { Meteorological } \\
\text { Observation }\end{array}$ & $\begin{array}{c}\text { Environmental } \\
\text { Monitoring }\end{array}$ \\
\hline Range [m] & $0-400$ & $0-1000$ & $0-5000$ & $0-10000$ & $0-15000$ \\
\hline Number of data & 13 & 19 & 45 & 70 & 150 \\
\hline Weighted logarithmic model [3] & $10.4 \%$ & $22.5 \%$ & $23.4 \%$ & $29.9 \%$ & $41.9 \%$ \\
Uniform distribution [9] & $12.6 \%$ & $18.1 \%$ & $29.7 \%$ & $\infty$ & $\infty$ \\
Exponential distribution [2] & $9.7 \%$ & $11.2 \%$ & $33 \%$ & $50 \%$ & $63.5 \%$ \\
Our method & $9 \%$ & $10.5 \%$ & $28.5 \%$ & $55.8 \%$ & $55.3 \%$ \\
\hline
\end{tabular}

Table 1. Mean relative errors of meteorological visibility distance estimation with respect to the envisioned applications on the MATILDA database.

sistent with the empirical method proposed previously by Babari et al. [3], which computes the temporal correlation between the variation of pixels intensity and the variation of the sky luminance estimated by an additional luminance meter oriented toward the North direction. By combining these two results, good experimental results are obtained which should simplify the calibration and the deployment of such camera-based visibility meters in the future.

\section{References}

[1] M. Andersen, T. Jensen, and C. Madsen. Estimation of dynamic light changes in outdoor scenes without the use of calibration objects. In International Conference on Pattern Recognition (ICPR'06), volume 4, pages 91-94, 2006.

[2] R. Babari, N. Hautière, E. Dumont, and N. Paparoditis. A model-driven approach to estimate atmospheric visibility with ordinary cameras. Atmospheric Environment, 2011.

[3] R. Babari, N. Hautière, E. Dumont, N. Paparoditis, and J. Misener. Visibility monitoring using conventional roadside cameras: Shedding light on and solving a multi-national road safety problem. In Transportation Research Board Annual Meeting Compendium of Papers, Washington, D.C., USA, 2011.

[4] D. Bäumer, S. Versick, and B. Vogel. Determination of the visibility using a digital panorama camera. Atmospheric Environment, 42:2593-2602, 2008.

[5] C. Bush and E. Debes. Wavelet transform for analyzing fog visibility. IEEE Intelligent Systems, 13(6):66-71, November/December 1998.

[6] CIE. International Lighting Vocabulary. Number 17.4. 1987.

[7] CIE. Spatial Distribution of Daylight - Luminance Distributions of Various Reference Skies. Number CIE 110-1994. 1994.

[8] N. Hautière, E. Bigorgne, J. Bossu, and D. Aubert. Meteorological conditions processing for vision-based traffic monitoring. In International Workshop on Visual Surveillance, European Conference on Computer Vision, 2008.

[9] N. Hautière, R. Babari, E. Dumont, R. Brémond, and N. Paparoditis. Lecture Notes in Computer Science, Computer Vi- sion - ACCV 2010, volume 6495, chapter Estimating Meteorological Visibility using Cameras: A Probabilistic ModelDriven Approach, pages 243-254. Springer, March 2011.

[10] S. Kim, J.-M. Frahm, and M. Pollefeys. Radiometric calibration with illumination change for outdoor scene analysis. In IEEE Computer Society Conference on Computer Vision and Pattern Recognition (CVPR'08), pages 1063-6919, 2008.

[11] S. Koppal and S. Narasimhan. Clustering appearance for scene analysis. In IEEE Computer Society Conference on Computer Vision and Pattern Recognition (CVPR'06), pages 1323 - 1330, 2006.

[12] J. Krochman. Über die horizontal Beleuchtungsstärke und die Zenitleuchtdichte des klaren Himmels. Lichttechnik, B22:551-554, 1970.

[13] J.-F. Lalonde, S. Narasimhan, and A. A. Efros. Lecture Notes in Computer Science, Computer Vision - ECCV 2008, volume 5305, chapter What Does the Sky Tell Us about the Camera?, pages 354-367. Springer, 2008.

[14] J.-J. Liaw, S.-B. Lian, Y.-F. Huang, and R.-C. Chen. Using sharpness image with haar function for urban atmospheric visibility measurement. Aerosol and Air Quality Research, 10:323-330, 2010.

[15] C.-H. Luo, C.-Y. Wen, C.-S. Yuan, C.-C. Liaw, J.-L. ans Lo, and S.-H. Chiu. Investigation of urban atmospheric visibility by high-frequency extraction: Model development and field test. Atmospheric Environment, 39:2545-2552, 2005.

[16] W. Middleton. Vision through the atmosphere. University of Toronto Press, 1952.

[17] S. G. Narasimhan and S. K. Nayar. Contrast restoration of weather degraded images. IEEE Transactions on Pattern Analysis and Machine Intelligence, 25(6):713-724, June 2003.

[18] R. Perez, R. Seals, and J. Michalsky. All-weather model for sky luminance distribution preliminary configuration and validation. Solar Energy, page 235 245, March 1993.

[19] A. Torralba and A. Oliva. Depth estimation from image structure. IEEE Transactions on Pattern Analysis and Machine Intelligence, 24(9):1-13, September 2002. 